\title{
Ensino em Saúde LGBT na Pandemia da Covid-19: Oportunidades e Vulnerabilidades
}

\author{
LGBT Health education during the Covid-19 Outbreak: Opportunities and Vulnerabilities
}

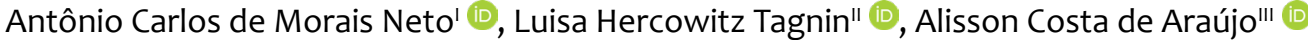

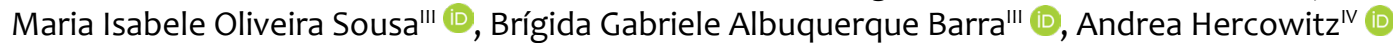

\section{RESUMO}

Introdução: A pandemia da Covid-19 e as medidas sanitárias de isolamento social impuseram a necessidade de reestruturação do ensino, com migração para tecnologias digitais. Em relação à educação em saúde da população de Lésbicas, Gays, Bissexuais, Travestis e Transexuais (LGBT), assunto que ainda está em processo de inserção nas escolas médicas, tal mudança trouxe novas oportunidades para a discussão do conteúdo, mas também criou e escancarou vulnerabilidades preexistentes.

Relato de Experiência: Ocorreram no Brasil diversas experiências de educação em saúde LGBT durante o período da pandemia da Sars-Cov-2, com auxílio de plataformas digitais. Em uma universidade do Rio Grande do Norte, uma disciplina optativa de Atenção à Saúde da População LGBT, que já seria ministrada no formato presencial, sofreu modificações e acabou por ser ofertada no modelo remoto. A liga de semiologia de uma faculdade de São Paulo se viu obrigada a mudar a sua aula com a mesma temática em decorrência do isolamento social.

Discussão: Essas experiências permitiram a análise de uma série de oportunidades e vulnerabilidades trazidas por esse momento de reformulações no ensino. A modalidade remota expôs e expandiu desigualdades sociais por conta da necessidade de equipamentos e internet para acesso aos conteúdos, marginalizando uma parcela vulnerável da população. Além disso, o ambiente digital pode ser inseguro para o pronunciamento de pessoas LGBT. Em contrapartida, essa modalidade permitiu a ampliação do público atingido pelas atividades, resultante da diminuição dos custos e da quebra de barreiras geográficas permitidas pelo ambiente digital. Surgiram inovações nas ferramentas de ensino, como uso de podcasts e vídeos, flexibilizando as formas de ensino e divulgação de informações.

Conclusão: Diante das deficiências encontradas com a experiência do ensino remoto emergencial, espera-se que, no futuro, os aprendizados adquiridos levem a uma implementação curricular mais democrática de atividades inclusivas em ensino sobre saúde LGBT nas universidades.

Palavras-chave: Educação Médica; Pessoas LGBT; Covid-19; Educação On-line.

\begin{abstract}
Introduction: The COVID-19 pandemic, and sanitary and social isolation measures imposed the need for restructuring health education, by migrating environments to digital technologies. As for health education for the Lesbian, Gay, Bisexual, Transvestite and Transsexual (LGBT) population, a subject that is still in the process of being inserted in medical schools, this change brought new opportunities for discussion, but it also created and brought up pre-existing vulnerabilities.

Experience Report: Around the country, during the Sars-Cov-2 pandemic period, there were several digitally-aided experiences of LGBT health education. At a University in Rio Grande do Norte, a non-mandatory discipline of Health Care for the LGBT Population, designed to be taught in a physical format, underwent modifications and ended up being offered remotely. The Semiology League at a College in São Paulo (SP) was forced to change its class with the same theme due to social isolation. In these and other experiences there were great challenges, but also a lot of innovation.

Discussion: Those experiences brought into focus a series of opportunities and vulnerabilities in this moment of educational changes. Remote education exposed and expanded social inequalities due to the need for equipment and internet access, marginalizing a vulnerable portion of the population. Furthermore, the digital environment proved not to be a totally safe setting for LGBT people to speak their minds. In contrast, this method allowed for the expansion of the audience in some activities, resulting from digital environment cost reductions and lower geographical barriers. Also, there were expanded innovations in teaching tools, such as the use of podcasts and videos, making teaching and information dissemination processes more flexible.
\end{abstract}

Conclusion: In view of the difficulties posed during these remote learning experiences, it is expected that, in the future, the lessons learned will lead to a more democratic curricular implementation of inclusive LGBT health activities in Universities.

Keywords: Medical Education; LGBT Persons; Covid-19; On-line Education.

'Universidade Federal do Rio Grande do Norte, Natal, Rio Grande do Norte, Brasil.

"Pontifícia Universidade Católica de São Paulo, São Paulo, São Paulo, Brasil.

"'Escola Multicampi de Ciências Médicas - Universidade Federal do Rio Grande do Norte, Caicó, Rio Grande do Norte, Brasil.

IV Faculdade Israelita de Ciências da Saúde Albert Einstein, São Paulo, São Paulo, Brasil.

Correspondência

Antônio Carlos de Morais Neto.

Avenida Passeio dos Girassóis, 2570, ap. 5, Natal, RN, Brasil. CEP: 59078-190.

E-mail: moraesneto123@gmail.com

Recebido em 09/08/20; Aceito em 14/09/20. 


\section{INTRODUÇÃO}

A população de Lésbicas, Gays, Bissexuais, Travestis e Transexuais (LGBT) historicamente e ainda hoje é alvo de preconceitos. Desde 1984 a homossexualidade vem sendo retirada dos manuais de doenças mentais por entidades médicas e governamentais. Aos poucos e com muita luta, começam a surgir políticas públicas, ações e programas voltados para essa população que visam atender às suas demandas específicas ${ }^{1,2}$. A Política Nacional de Saúde Integral de Lésbicas, Gays, Bissexuais, Travestis e Transexuais (PNSI LGBT), publicada em 2012, foi um grande passo em busca de maior equidade do Sistema Único de Saúde (SUS), com o objetivo de promover saúde integral da população LGBT, eliminando discriminação e preconceito institucional e reduzindo desigualdades 3 .

Contudo, pesquisas mostram que essa população tem menor acesso ao sistema de saúde, o que, em grande parte, deve-se ao atendimento não humanizado, discriminatório e resistente às questões de diversidade sexual por parte de profissionais da saúde ${ }^{4,5}$. Esses dados refletem o desconhecimento desses profissionais em relação às especificidades das queixas da população LGBT e do atendimento a ela ${ }^{6,7}$, resultante da lacuna curricular das escolas médicas e de outras áreas da saúde na abordagem dessas questões. Portanto, é essencial que haja capacitação para o atendimento integral e humanizado de pessoas LGBT tanto em cursos de graduação, conforme os artigos $5^{\circ}$ e 12 das Diretrizes Curriculares Nacionais do Curso de Graduação em Medicina (DCN) de 2014, quanto em pós-graduação e até mesmo dentro de instituições destinadas à prática da saúde ${ }^{8,9}$.

A pandemia causada pela síndrome respiratória aguda grave do coronavírus 2 (severe acute respiratory syndrome coronavirus 2 - Sars-CoV-2) - coronavirus disease 2019 (Covid-19) - alterou a economia global, as relações interpessoais e de trabalho e o sistema educacional, exigindo o fechamento temporário das escolas e instituições de ensino superior $^{10}$. Ante a adoção de medidas de combate à pandemia, como a suspensão das atividades letivas presenciais, ocorreu a migração de professores e estudantes para a realidade online, fruto da necessidade de reestruturação do ensino ${ }^{11}$. A transposição de metodologias e práticas educativas típicas para a realidade virtual é designada como ensino remoto de emergência"1, com a adoção desse sistema de ensino por muitas instituições de ensino superior do país. No entanto, as tecnologias de informação e comunicação já estavam presentes nas estratégias educacionais como ferramentas facilitadoras no processo de aprendizagem do aluno ${ }^{12}$.

Por conta das circunstâncias da pandemia, mais do que transferir as práticas presenciais, é importante criar ambientes de aprendizagem colaborativa nas plataformas escolhidas para o ensino remoto ${ }^{11}$. Ensinar de forma remota não é o mesmo que ensino a distância, embora ambos tenham relação direta com o uso da tecnologia digital. Um curso criado especialmente para ser acessado on-line envolve formas assíncronas de contato entre alunos e instrutores, e diferentes instrumentos de avaliação. O formato remoto comporta outras potencialidades e introduz desafios que envolvem o sincronismo da presença de alunos e instrutores, expertise e tecnologia e infraestrutura mais robustas $^{13}$. Entre as maiores dificuldades dessa modalidade de ensino, está a exclusão de alunos que não têm acesso à internet ou aos equipamentos necessários para que possam acompanhar as atividades ${ }^{14-16}$. Observa-se que a situação produzida pela Covid-19 evidenciou novas vulnerabilidades e intensificou as já existentes no ensino presencial ${ }^{17}$. Apesar disso, esse momento ímpar de mudanças educacionais trouxe experiências positivas de expansão das possibilidades.

O objetivo deste relato é descrever experiências de educação de saúde LGBT no formato de ensino remoto emergencial, analisando as possibilidades e vulnerabilidades encontradas no processo.

\section{RELATO DE EXPERIÊNCIA}

Em uma universidade no sertão do Rio Grande do Norte, existia a disciplina optativa de Atençãoà Saúde da População LGBT que, em seu formato presencial, antes da adoção das medidas de distanciamento social, contava com 21 alunos matriculados. Com a pandemia do novo coronavírus, surgiu a proposta de ofertála no modelo remoto de ensino, o que, a princípio, foi encarado como um grande desafio e com baixas expectativas pelo corpo docente. Porém, o modelo remoto permitiu que, pela primeira vez na instituição, barreiras burocráticas fossem rompidas e a matrícula de alunos de outros câmpus e cursos se tornasse possível. Formou-se então um grupo de 50 alunos de Medicina e de outros cursos, como Psicologia, Enfermagem, Educação Física e Direito. Além disso, esses estudantes eram de cidades e orientações sexuais e de gênero diferentes.

A disciplina, inspirada nas ideias da educação popular em saúde e em metodologias participativas, necessitou de uma reorganização para existir naquele espaço virtual. Foram realizadas atividades síncronas, duas vezes por semana, por meio da plataforma Google Meets. Além disso, semanalmente, os alunos precisavam fazer comentários nos fóruns do site da universidade sobre suas compreensões dos conceitos apresentados em aula aliados a materiais indicados pelo docente, como textos, vídeos e filmes.

Dos muitos desafios encontrados nesse processo, o primeiro foi fazer um grupo diverso se enxergar como coletivo. No entanto, essa situação foi superada ainda nos primeiros encontros, de forma gradual, com a apresentação 
dos estudantes e o incentivo ao compartilhamento de suas vivências. Isso transformou as discussões em espaços de troca de experiências e também de aprendizado interprofissional.

Alguns alunos relataram problemas em acompanhar as aulas de forma eficiente, por terem uma conexão lenta à internet ou por estarem em locais afastados de um sinal adequado. A dificuldade de garantir que as ferramentas estivessem funcionando influenciava a participação nos encontros. Mesmo assim, conseguiu-se, na medida do possível, um espaço de fala para todos, com a gravação das aulas e a participação pelo chat, que não necessitava desses instrumentos em pleno funcionamento.

A criação de um espaço virtual seguro para os estudantes possibilitou, por meio do diálogo e da escuta, a formação de vínculo entre os membros da disciplina e a participação ativa dos discentes. Os alunos LGBT ficaram confortáveis para se posicionar como parte de um grupo minoritário e tiveram suas vozes ouvidas. Isso foi de grande ajuda para esses acadêmicos que, por causa da pandemia, estão em tempo integral na casa de suas famílias, que pode ser um ambiente pouco saudável para pessoas LGBT. Dessa maneira, a relação tradicional entre um aluno e uma disciplina foi ressignificada para uma relação de responsabilidade e compartilhamento de histórias, afetos e aprendizados.

A disciplina também tinha a proposta de trazer para momentos de fala convidados da comunidade LGBT, assim como dar representatividade aos movimentos sociais locais. Todos os encontros contaram com a presença de um representante de movimentos sociais locais, com a finalidade de trazer uma perspectiva social e política diferente do olhar biomédico. Porém, uma barreira encontrada nessa intenção foi a necessidade de uma estrutura tecnológica mínima exigida pelo modelo remoto, bem como a habilidade para o uso desses equipamentos. Logo, as pessoas LGBT em maiores situações de vulnerabilidade foram impossibilitadas de participar desses momentos.

Apesar dessas dificuldades, estiveram presentes vários convidados de diversos estados do país, que possuíam autoridade nos assuntos de saúde LGBT. Assim, a criação de um grupo aliado ao ensino remoto possibilitou não só a quebra da barreira relacionada à questão identitária dos discentes e do espaço, mas também da barreira geográfica entre os convidados e os alunos. O contato com o grupo de estudo foi enriquecedor, partindo do pressuposto da troca ativa de informações e de percepções subjetivas relacionadas à temática.

Com o apoio dos diversos convidados, os tópicos debatidos criaram um olhar plural, envolvendo as múltiplas facetas da história, das condições e da necessidade desse grupo de pessoas. Assim, os assuntos envolviam as definições dentro da sigla LGBT, o processo transexualizador na atenção básica, a Política de Saúde Integral LGBT do SUS, a saúde mental de pessoas LGBTs, o acesso integral em saúde por transexuais e vulnerabilidades, como a utilização de silicone industrial, os exames ginecológicos para o homem trans e a mulher lésbica e as principais demandas no atendimento médico a essa população. Além disso, buscando um olhar mais humano a respeito dessa questão, abordou-se a história dos movimentos sociais LGBTs, a hormonioterapia através de um olhar antropológico e as vivências de pessoas trans, narradas por essas pessoas.

Sem perder a proposta de a disciplina existir também para fora dos muros da universidade, e assim ressignificar os espaços acadêmicos, por vezes tão arriscados para pessoas LGBT, foram usadas estratégias de divulgação de conteúdos e experiências nas redes sociais. Os principais recursos para esse contato com a população foram postagens com divulgação do trabalho de artistas locais, pertencentes à comunidade, e infográficos sobre temas de interesse do público-alvo, como dicas de filmes, séries e conteúdos, incluindo a questão do nome social e a situação LGBT na pandemia atual.

Uma das formas de avaliação do componente curricular foi a construção de produtos que realizassem algum retorno para a sua comunidade-alvo. Pela realização da disciplina em formato remoto, as produções ultrapassaram as expectativas e simbolizaram um resultado disponível no ambiente virtual, mostrando uma forma possível de alcançar uma parte da população LGBT com o uso das tecnologias. Os produtos desse processo foram a fabricação de cartilhas e vídeos educativos sobre especificidades da saúde LGBT e um programa de podcast para discussão longitudinal dos temas da disciplina.

Outras experiências com objetivo de discutir a temática aconteceram pelo país durante esse mesmo período. A liga de semiologia de uma faculdade de São Paulo organizou a palestra inaugural com a temática em saúde LGBT para março de 2020, com uma expectativa de presença de cerca de 30 alunos. No entanto, esse foi justamente o momento em que todas as instituições de ensino foram fechadas, e as aulas presenciais, canceladas. Após uma pausa para reorganização, foi reagendada para abril, via plataforma digital. A palestra foi apresentada por meio da plataforma Google Meets, com o título "Diversidade sexual: compreender para melhor acolher". Houve uma diferença marcante de público, estando presentes 160 pessoas, de diversas faculdades de Medicina do estado de $\mathrm{SP}$, número bem maior do que o esperado.

A ideia era ampliar o conhecimento sobre a diversidade sexual e sensibilizar estudantes de Medicina para um acolhimento adequado da população LGBT Inicialmente, definiram-se os termos e explicou-se a sigla. A seguir, abordaram-se os seguintes assuntos: formação da orientação sexual e da identidade de gênero; quem são as pessoas 
intersexo; barreiras de acesso e principais agravos de saúde de cada subgrupo dessa população; como criar um ambiente de atendimento inclusivo, com treinamento de toda a equipe do local; o papel do profissional da saúde e como devem ser abordadas as pessoas dessa comunidade em cada período da vida, com dicas de uma anamnese acolhedora e exame físico respeitoso; a Resolução do Conselho Federal de Medicina (CFM) nº 2.265/2019; e especificidades do acompanhamento de pessoas transgênero. $O$ assunto foi bem recebido pelos ouvintes, havendo participação ativa com dúvidas durante a apresentação e discussão no final. Por meio desse contato, convites para palestras em outras universidades surgiram, ampliando as possibilidades de atingir um número ainda maior de estudantes.

\section{DISCUSSÃO}

A importância da introdução da discussão de identidade de gênero, orientação sexual e sexualidade nas escolas médicas já está bem esclarecida, e, apesar de na prática muitos cursos ainda não integrarem o assunto, ele já foi inserido na DCN de graduação em Medicina. Um estudo que analisou os projetos pedagógicos curriculares (PPC) de escolas médicas federais no Brasil demonstrou que mais de $50 \%$ deles abordam os temas gênero e/ou sexualidade ${ }^{18}$. No entanto, essa e outras pesquisas, do ponto de vista de docentes e discentes, reiteram que o conteúdo abordado geralmente tem enfoque nos aspectos biológicos e patológicos, deixando de lado a parte psicossocial que tem grande influência no processo saúdeadoecimento-cuidado da população $\mathrm{LGBT}^{8,19}$.

\section{Oportunidades}

A ampliação do público inscrito na disciplina de Saúde LGBT no Rio Grande do Norte e na palestra inaugural da liga de semiologia em São Paulo é decorrente da maior flexibilidade proporcionada pelo ambiente de ensino on-line, que não apresenta restrição espacial. Essa nova forma de contato com o conhecimento amplia a possibilidade de aprendizagem e o envolvimento entre os atores do processo ${ }^{20}$. As novas tecnologias incorporadas permitem que barreiras geográficas e temporais sejam ultrapassadas, amplificando as possibilidades de colaboração ${ }^{21}$. O ensino remoto e os eventos on-line criam uma comunidade virtual que proporciona conexões entre pessoas de diversos lugares do país e do mundo, formando uma rede colaborativa entre indivíduos diferentes para alcançar objetivos comuns ${ }^{22}$.

As inovações utilizadas para superar os desafios enfrentados pelas organizações e instituições de ensino com a pandemia encontram aspectos positivos no que concerne à burocracia e ao custo de suas atividades. Os cursos, simpósios, congressos e outros eventos on-line têm custo substancialmente reduzido com a hospedagem em plataformas de nuvem ${ }^{22}$. Gastos com aluguel de espaço e organização do local, e a burocracia para a reserva de salas em universidades dificultam a realização de muitos eventos. Outro benefício percebido por professores e palestrantes é a redução do tempo gasto fora de suas casas, minimizando os custos e aumentando sua produtividade ${ }^{22-4}$.

O perfil do graduando em Medicina, baseado nas últimas DCN, possui características voltadas para um empoderamento dos estudantes no processo de aprendizagem e de estímulos para inovações na formação9. Nesse sentido, a situação ocasionada pela pandemia e a necessidade de um ensino remoto emergencial proporcionaram diversas adaptações para o ensino nas escolas médicas pelo mundo ${ }^{23}$. As teleconferências tornaram-se a principal tecnologia utilizada para disseminar o conhecimento e possibilitar um aprendizado interdisciplinar. A oferta de disciplinas e a realização de webinars e eventos on-line neste período de isolamento proporcionam um ambiente eficaz para inovações e possíveis legados para a educação médica²3.

Outro aspecto ressaltado nas DCN é a formação ética e crítica com responsabilidade social nos âmbitos individuais e coletivos 9 . Nesse sentido, a discussão sobre saúde LGBT dentro da graduação de Medicina exige um reconhecimento das vulnerabilidades dessa comunidade e uma reflexão sobre alternativas para superá-las. Na tentativa de alcançar essa população, as plataformas digitais e as mídias sociais provam ser duas das principais formas de acesso à informação para as pessoas $\mathrm{LGBT}^{24}$, pois simbolizam uma forma de conseguir cumprir seus objetivos de aprendizado, de forma anônima e sem estigmatização, e oferecem locais de encontro entre pessoas que passam por situações similares ${ }^{25}$. Esses ambientes criam uma corrente de transmissão de informação, transformando os espectadores em produtores ${ }^{25}$ de conteúdo.

As tecnologias digitais têm sido importantes aliadas no acesso à educação médica, sendo utilizadas como ferramenta para disseminação de informações. Um exemplo disso são os podcasts, áudios previamente utilizados para entretenimento e notícias, que agora são uma potencial alternativa para o aprendizado individual de conteúdo científico, com flexibilização dos espaços de ensinar e aprender ${ }^{23,26}$.

A realidade das universidades para a comunidade LGBT muitas vezes carrega um preconceito que interfere no ambiente acadêmico desses estudantes ${ }^{27}$. Durante a disciplina ministrada no Rio Grande do Norte, foi construído um ambiente que foge da maioria dos cenários no Brasil, possibilitando a formação de um coletivo de confiança e um local identitário e representativo para os alunos LGBT. A construção de uma identidade relaciona-se com condições e contextos sociais do indivíduo, que o incluem ou excluem de um ambiente, 
seja um bar ou uma universidade ${ }^{28}$. Esse processo viabiliza o posicionamento dos indivíduos permitindo que suas vozes sejam ouvidas. Nesse sentido, a experiência do componente virtual possibilitou a construção de um local de representação para os alunos dentro da universidade, criando identidades individuais e coletivas relacionadas aos temas em debate ${ }^{29}$.

\section{Vulnerabilidades}

O ensino remoto apresenta uma série de dificuldades intrínsecas, como o acesso à internet e a equipamentos necessários para o aprendizado. Problemas técnicos com tais equipamentos são muito comuns e afetam diretamente o aproveitamento das aulas. Além disso, de acordo com a Organização das Nações Unidas para a Educação, a Ciência e Cultura (Unesco), um dos pontos mais críticos no Brasil em relação à internet é a desigualdade das condições de uso e posse de tecnologias de informação e comunicação ${ }^{30}$. A Pesquisa Nacional por Amostra de Domicílios Contínua (Pnad Contínua) do Instituto Brasileiro de Geografia e Estatística (IBGE) mostra que um em cada quatro brasileiros não possui acesso à internet e que um dos equipamentos que poderiam proporcionar o acesso às aulas, o microcomputador, está presente em apenas $48,1 \%$ dos lares brasileiros ${ }^{31}$. Dessa forma, o ensino remoto tende a reforçar as desvantagens sociais, marginalizando populações mais vulneráveis e excluindo-as do aprendizado.

Ao abordar temas como saúde LGBT, é fundamental que o docente consiga interagir com os discentes, observar suas reações e criar vínculo, tanto para perceber distrações e atraí-los quanto para acolher aqueles que necessitam. Aulas on-line dificultam essa dinâmica, pois, diferentemente das presenciais, em que é possível analisar comportamentos e ações dos alunos e criar diálogos, a interação é mais superficial. A relação entre discentes e docentes é ainda mais prejudicada por conta da tendência a deixar as câmeras desligadas (no caso daqueles que possuem esse equipamento) e pelo fato de que, em muitas plataformas digitais utilizadas para o ensino, o professor, ao compartilhar a tela, é impossibilitado de visualizar os alunos. Outro aspecto que dificulta o aprendizado é a falta de capacitação prévia dos docentes, pois isso exige uma dinâmica de aula diversificada que consiga prender a atenção dos estudantes e passar o conteúdo de maneira adequada.

Além disso, no ensino de saúde específico de uma comunidade, é essencial a presença de pessoas que pertencem a ela, permitindo o compartilhamento de experiências e discussões dos aspectos humanitários. No entanto, a experiência de ser LGBT em uma sociedade polarizadora e desigual leva essa população a situações de vulnerabilidade, como trabalhos informais e falta de ambientes protegidos. Isso pode dificultar o cumprimento do isolamento social, aumentando o risco de contágio de Covid-19, além de exacerbar riscos adicionais de morbidades decorrentes de infecções sexualmente transmissíveis, HIV, abuso de drogas, desordens psiquiátricas e suicídio ${ }^{32}$. Os prejuízos na saúde física e mental desses indivíduos influenciam diretamente seu processo de aprendizagem e inibem sua presença em ambientes de educação em saúde LGBT, sejam estes presenciais ou remotos, empobrecendo essa discussão para todos. Um ambiente seguro e inclusivo à diversidade sexual e de gênero, sem violências motivadas pelo preconceito, favorece o senso de comunidade e, consequentemente, o aprendizado de pessoas LGBT. Criar o mesmo ambiente por meio de aulas on-line no momento de isolamento social pode tornar-se impossível para estudantes LGBT que vivem em lares LGBTfóbicos. O constrangimento e o medo de se exporem ao participarem de atividades que abordem orientação sexual e identidade de gênero, quando não estão em locais privativos, excluem essas pessoas, tirando-lhes a voz e impedindo um bom aproveitamento.

Assim como há um desafio de tornar o espaço físico mais seguro, há também a problemática do espaço virtual ser seguro, uma vez que o docente não tem poder para controlar esses lugares no modelo de ensino remoto emergencial. Ali, não há monitoração adequada acerca da gravação do conteúdo, de quem está assistindo e das mensagens trocadas entre os participantes. São comuns algumas práticas ${ }^{33} \mathrm{e}$ ataques de ódio virtuais, violações por múltiplos ataques, fake news ${ }^{34}$, invasões de hackers ou até violências imperceptíveis e simbólicas presentes na comunicação, que podem tanto ser direcionadas aos alunos, à matéria, ao evento ou aos docentes, com a intenção de caluniar ou promover um linchamento virtual ${ }^{35}$.

\section{CONCLUSÃO}

Em meio à pandemia de Covid-19, o ensino remoto trouxe muitas oportunidades, porém apontou algumas vulnerabilidades para a educação em saúde LGBT. O ambiente virtualimpôsobstáculos comoa diminuição dainteraçãoentreos discentes e docentes, possíveis problemas técnicos, dificuldade de acesso e inclusão para pessoas em maior vulnerabilidade, e ausência de um ambiente protegido para alunos em situações particulares. Entretanto, houve vantagens significativas que permitiram uma ampliação do público e do acesso por conta tanto da quebra das barreiras geográficas entre os discentes e profissionais de referência na área, quanto da possibilidade de divulgação em massa oferecida por tecnologias digitais, além da diminuição do custo, da burocracia e da resistência em se fazerem eventos que pensem nesse tema. Dessa maneira, a criação de disciplinas e eventos que discutam essa temática 
torna-se uma possibilidade, ao fornecer meios flexíveis para a divulgação de informações e contornar algumas barreiras e entraves a esse tipo de conteúdo. Espera-se que, no futuro, os aprendizados adquiridos com essas experiências no ensino remoto emergencial levem a uma implementação curricular mais democrática de atividades inclusivas em ensino sobre saúde LGBT nas universidades e que os fatores causais das vulnerabilidades possam ser minimizados.

\section{CONTRIBUICุÃO DOS AUTORES}

Todos os autores contribuíram igualmente para a produção do artigo, sob a orientação das professoras Andrea Hercowitz e Brígida Gabriele Albuquerque Barra. A revisão final do artigo coube à professora Andrea Hercowitz.

\section{CONFLITO DE INTERESSES}

Os autores declaram não haver conflito de interesses neste relato.

\section{REFERÊNCIAS}

1. Laurentino ACN. Políticas públicas de saúde para população LGBT: da criação do SUS à implementação da Política Nacional de Saúde Integral de LGBT [dissertação]. Rio de Janeiro: Escola Politécnica de Saúde Joaquim Venâncio, Fundação Oswaldo Cruz; 2015.

2. Organização Mundial da Saúde. Classificação de transtornos mentais e de comportamento da CID - 10: descrições clínicas e diretrizes diagnósticas. Porto Alegre: Artes Médicas; 1998.

3. Brasília. Política Nacional de Saúde Integral de Lésbicas, Gays, Bissexuais, Travestis e Transexuais. Brasília: Ministério da Saúde; 2013 [acesso em 16 set 2020]. Disponível em: http://bvsms.saude.gov.br/bvs/publicacoes/ politica_nacional_saude_lesbicas_gays.pdf.

4. Bittencourt $D$, Fonseca $V$, Segundo M. Acesso da população LGBT moradora de favelas aos serviços públicos de saúde: entraves, silêncios e perspectivas. Conexões Psi. 2015;12;2(2):60-85.

5. Soinio JI, Paavilainen E, Kylmä JP. Lesbian and bisexual women's experiences of health care: “Do not say,'husband', say,'spouse”'. J Clin Nurs. 2020;29(1-2):94-106.

6. Cardoso MR, Ferro LF. Saúde e população LGBT: demandas e especificidades em questão. Psicol Ciênc. Prof. 2012;32(3):552-63.

7. Negreiros FR, Ferreira BD, Freitas DD, Pedrosa JI, Nascimento EF. Saúde de lésbicas, gays, bissexuais, travestis e transexuais: da formação médica à atuação profissional. Rev Bras Educ Med. 2019;43(1):23-31.

8. Rufino AC, Madeiro AP, Girão MJ. O ensino da sexualidade nos cursos médicos: a percepção de estudantes do Piauí. Rev Bras Educ Med. 2013;37(2):178-85.

9. Brasil. Resolução $\mathrm{n}^{\circ} 03$, de 20 de junho de 2014. Institui Diretrizes Curriculares Nacionais do Curso de Graduação em Medicina e dá outras Providências.

10. Alves BAL. Letramento digital em tempos de COVID-19: uma análise da educação no contexto atual. Debates em Educação. 2020;12(28):1-18.

11. Moreira JA, Henriques S, Barros DM. Transitando de um ensino remoto emergencial para uma educação digital em rede, em tempos de pandemia. Dialogia. 2020:351-64.

12. Koch MZ. As tecnologias no cotidiano escolar: uma ferramenta facilitadora no processo de ensino- aprendizagem [monografia]. Sarandi: Universidade Federal de Santa Maria; 2013.
13. Garcia TCM, Morais IRD, Zaros LG, Rêgo MCFD. Ensino remoto emergencial: proposta de design para organização de aulas [monografia]. Natal: Universidade Federal do Rio Grande do Norte; 2020.

14. Torres ACM, Alves LRG, da Costa ACN. Education and health: reflections on the university context in times of COVID-19. SciELO Preprints; 2020. doi: $10.1590 /$ SciELOPreprints.640.

15. Centro Regional de Estudos para o Desenvolvimento da Sociedade da Informação. TIC Domicílios 2019 [acesso em 7 ago 2020]. Disponível em: https://cetic.br/media/analises/tic_domicilios_2019_coletiva_imprensa.pdf.

16. Sampaio C. Professores, pais e alunos apontam dificuldades e limitações do ensino a distância. Brasil de Fato; 4 maio 2020 [acesso em 7 ago 20]. Disponível em: https://www.brasildefato.com.br/2020/05/04/professorespais-e-alunos-apontam-dificuldades-e-limitacoes-no-ensino-a-distancia.

17. Vieira L, Ricci MCC. A educação em tempos de pandemia: soluções emergenciais pelo mundo . Observatório do Ensino Médico em Santa Catarina; abr 2020 [acesso em 7 de ago 2020]. Disponível em: https://www. udesc.br/arquivos/udesc/id_cpmenu/7432/EDITORIAL_DE_ABRIL__Let cia_Vieira_e_Maike_Ricci_final_15882101662453_7432.pdf.

18. Raimondi GA, Abreu YR, Borges IM, Silva GB, Hattori WT, Paulino DB. Gênero e sexualidade nas escolas médicas federais do Brasil: uma análise de projetos pedagógicos curriculares. Rev Bras Educ Med. 2020;44(2):e046.

19. Rufino AC, Madeiro A, Girão MJ. Sexuality education in Brazilian medical schools. J Sex Med. 2014;11(5):1110-7.

20. de Oliveira GP. O fórum em um ambiente virtual de aprendizado colaborativo. São Paulo: Associação Brasileira de Educação a Distância; 2011.

21. de Barros ÁG, de Souza CH. A internet de todas as coisas e a educação: possibilidades e oportunidades para os processos de ensino e aprendizagem. Linkscienceplace. 2017 Apr 5;3(3):31-45.

22. Appana S. A review of benefits and limitations of online learning in the context of the student, the instructor and the tenured faculty. International Journal on E-learning. 2008;7(1):5-22.

23. Dedeilia A, Sotiropoulos MG, Hanrahan JG, Janga D, Dedeilias P, Sideris $M$. Medical and surgical education challenges and innovations in the COVID-19 era: a systematic review. In Vivo. 2020;34(3 Suppl):1603-11.

24. Gorett M, Grossi R, Murta FC, Silva MD. A aplicabilidade das ferramentas digitais da Web 2.0 no processo de ensino e aprendizagem. Rev Context Educ. 2018;33(104):34-59. doi: 10.21527/2179-1309.2018.104.34-59.

25. Fox J, Ralston R. Queer identity online: Informal learning and teaching experiences of LGBTQ individuals on social media. Comput Human Behav. 2016;65:635-42. doi: 10.1016/j.chb.2016.06.009.

26. Tiago TS, Minuzi N, Barin CS, Araújo LM. A utilização do podcast como uma ferramenta inovadora no contexto educacional. Redin - Revista Educacional Interdisciplinar. 2018;7(1) [acesso em 7 de ago de 2020]. Disponível em: https://seer.faccat.br/index.php/redin/article/view/1143.

27. dos Santos JB. A condição de ser LGBT e a permanência na universidade: um estudo de caso no curso de pedagogia - educação do campo. Universidade Federal da Paraíba; 2017 [acesso em 3 ago 2020]. Disponível em: http:// plone.ufpb.br/nepes/contents/documentos/trabalhos-publicados-emanais/a-condicao-de-ser-lgbt-e-a-permanencia-na-universidade-um-estudode-caso-no-cuso-de-pedagogia-educacao-do-campo.pdf/view.

28. Woodward K. Identidade e diferença: uma introdução teórica e conceitual. Identidade e diferença: a perspectiva dos estudos culturais. Petrópolis: Vozes; 2000 [acesso em 7 de ago de 2020]. Disponível em: https://edisciplinas.usp.br/pluginfile.php/4284077/mod_resource/ content/1/cap\%C3\%ADtulo\%20l\%20-\%20Woodward\%20-\%20IDENTIDADE-EDIFERENCA-UMA-INTRODUCAO-TEORICA-E-CONCEITUAL.pdf.

29. Souza MH. A Militância LGBT na universidade: um estudo de caso do Coletivo KIU [dissertação]. Salvador: Universidade Federal da Bahia; 2015 [acesso em 7 de ago de 2020]. Disponível em: http://repositorio.ufba.br/ ri/handle/ri/18172.

30. United Nations Educational, Scientific and Cultural Organization, Brazilian Network Information Center, Regional Centre of Studies for the Development of the Information Society. Assessing internet development in Brazil: using UNESCO's Internet Universality ROAM-X. 2019 [acesso em 7 ago 2020]. Disponível em: https://unesdoc.unesco.org/ ark:/48223/pfooo0372330. 
31. PNAD Contínua TIC 2018: Internet chega a 79,1\% dos domicílios do país. Agência IBGE notícias; 29 abr. 2020 [acesso em 7 ago 2020. Disponível em: https://agenciadenoticias.ibge.gov.br/agencia-sala-de-imprensa/2013agencia-de-noticias/releases/27515-pnad-continua-tic-2018-internetchega-a-79-1-dos-domicilios-do-pais?fbclid=IwAR3WmjmpS8Pdi7DOuyoD klgLRdulGDZ10Xy32J020Oj-uABUBGpBF-bADWY.

32. Banerjee D, Nair VS. "The Untold Side of COVID-19": struggle and perspectives of the sexual minorities. J Psychosexual Heal. $2020 \mathrm{Jul}$ 24;2(2):113-20. doi: 10.1177/2631831820939017.

33. de Carvalho FSP, Spineli P. Práticas ciberfascistas: é possível pensar-fazer uma engenharia reversa que rompa com o implante desejante fascista? Revista Communitas. 2020;4(7):43-58.
34. Braga RMC. A indústria das fake news e o discurso de ódio. In: Pereira $\mathrm{RV}$, organizador. Direitos políticos, liberdade de expressão e discurso de ódio. Belo Horizonte: Idde; 2018. v. 1, p. 203-20.

35. Lobo RAÁ, Coutinho Filho MSD. Notas sobre o linchamento em rede: apontamentos introdutórios a partir de uma pesquisa documental em andamento. Anais do IX Simpósio Nacional da ABCiber: Cibercultura, Democracia e Liberdade no Brasil; 2016. São Paulo: ABCiber; 2016. 